\section{The Photoelectron Spectra of Cyclopropyl Ketones}

Jabria A. Al-Khafaji and Muthana Shanshal

Department of Chemistry, College of Science, University of Baghdad, Adhamiya, Baghdad, Iraq

(Z. Naturforsch. 32 a, 109-110 [1977] ; received October 8, 1976)

The photoelectron spectra of 1- and 2-methyl-cyclopropyl ketones are reported. It is found that the shifts in the MO energies of $\Delta$ ext.as. and $\Delta$ ext.s. MO's of the three membered ring are parallel to the predictions based on the perturbation of the Walsh orbitals and on the MINDO/2 calculations.

The interaction of the cyclopropyl group with the unsaturated aliphatic moieties may be described in terms of a second order perturbation of the Walsh molecular orbitals (MO's) and the aliphatic $\pi$-MO's ${ }^{1}$. Theoretical perturbation treatments ${ }^{1}$ and experimental photoelectron spectroscopic measurements ${ }^{2}$ done for some aliphatic cyclopropyl derivatives showed that these interaction energies are of the magnitudes common to the aliphaltic-aliphatic interactions. The perturbation treatment enabled the explanation of the appearance of a $\Delta-\pi^{*}$ electronic absorption band in the spectra of cyclopropyl ketones ${ }^{3,4}$. To study the influence of the $\mathrm{C}=\mathrm{O}$ group on the energies of the Walsh MO's in the cyclopropyl ketones we have measured the PE spectra of the following molecules; (a) cyclopropylmethyl ketone, (b) 1-methyl-cyclopropyl-methyl ketone and (c) 2-methyl-cyclopropyl-methyl ketone. The obtained ionization potentials and the calculated MINDO/2 MO energies are discussed in the following text.

\section{Experimental}

Synthesis and analysis values of the ketones were published recently ${ }^{3}$. The PE spectra were recorded on a PS 18 instrument (Perkin Elmer Ltd.) at the Institut für Organische Chemie, T.H. Darmstadt, W.-Germany.

\section{Results and Discussion}

The 4 highest bands in the PE spectrum of cyclopropyl-methyl ketone correspond to the following ionization energies; $9.46 \mathrm{eV}, 10.58 \mathrm{eV}, 11.33 \mathrm{eV}$ and $12.64 \mathrm{eV}$. According to Koopmans theorem ${ }^{5}$

Reprint requests to Dr. Muthana Shanshal, Department of Chemistry, College of Sciences, University of Baghdad, Adamiya-Baghdad, Irak. we relate these bands to the following MINDO/2 MO's: n-MO $(9.53 \mathrm{eV}), \Delta_{\text {ext.as. }}(10.52 \mathrm{eV}), \Delta_{\text {ext.s. }}$ $(10.68 \mathrm{eV})$ and $\pi-\mathrm{MO}(10.62 \mathrm{eV})$ respectively (Figure 1). The order of the 1st 3 MO's is similar to that predicted recently on the basis of the perturbation treatment ${ }^{3}$.

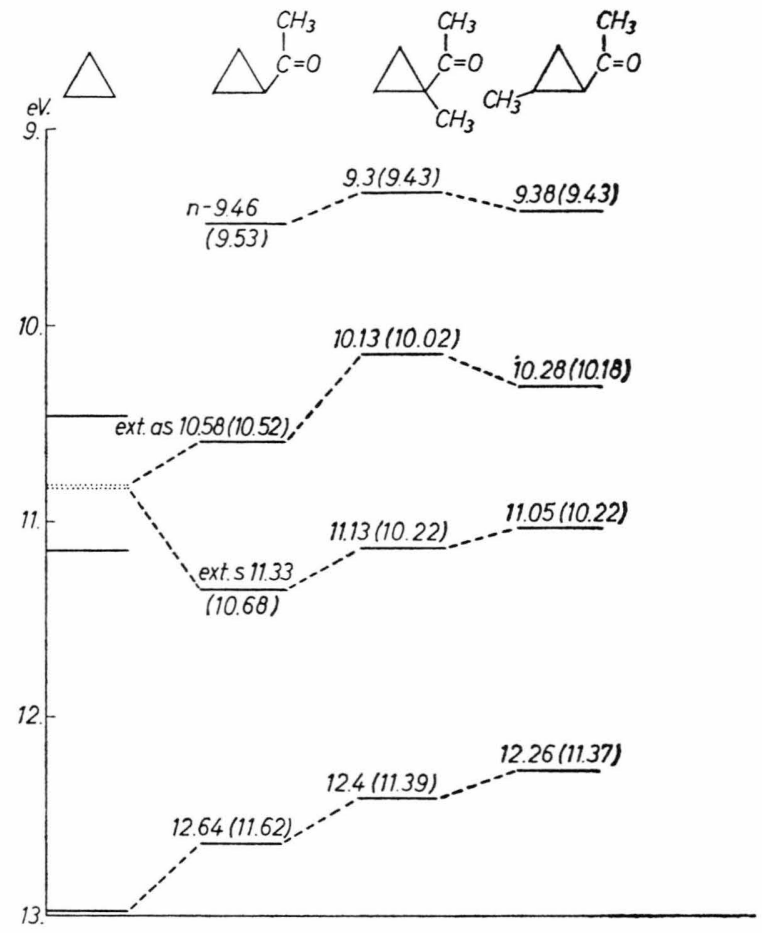

Fig. 1. RE ionization energies and MINDO/2 (MO energies) of cyclopropyl-methyl ketone; 1-methyl-cyclopropylmethyl ketone and 2-methyl-cyclopropyl-methyl ketone compared with the energies of cyclopropane.

The MINDO/2 calculations show that the n-MO is partly delocalized over the three membered ring. Its main contributor is the Op,z AO. The second higher, $\Delta_{\text {ext.as. }}$, MO is negatively combined with the $\mathrm{C}=\mathrm{O} \pi$-MO. The third, $\Delta_{\text {ext.s. }}$, MO is negatively combined with the $\mathrm{C}-\mathrm{O} \sigma$-MO. The $\pi$-MO extends over the $\mathrm{H}_{3} \mathrm{C}-\mathrm{CO}$ group. Figure 2 shows the distribution diagrams of the 4 MO's.

Introducing a methyl group to the $\mathrm{C}_{1}$ atom of the ketone causes an inductive shift in the energies of all 4 MO's. The new ionization energies (and MINDO/2 MO energies) are: n-MO $9.3 \mathrm{eV}(9.43$ $\mathrm{eV}), \Delta_{\text {ext.as. }} 10.13 \mathrm{eV}(10.02 \mathrm{eV}), \Delta_{\text {ext.s. }} 11.13 \mathrm{eV}$ $(10.22 \mathrm{eV})$ and $\pi-\mathrm{MO} 12.4 \mathrm{eV}(11.39 \mathrm{eV})$. The comparison with the MO energies of cyclopropylmethyl ketone shows that the $\Delta_{\text {ext.as. }}$ MO undergoes the biggest shift $(\Delta I=.45 \mathrm{eV})$ due to the methyl 


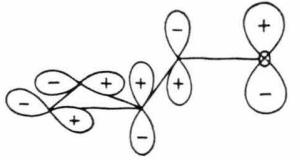

$\mathrm{n}-\mathrm{MO}$

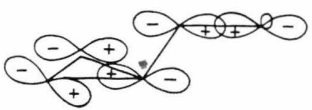

$\triangle$ ext.as.

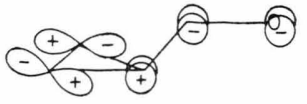

$\triangle$ ext.s.

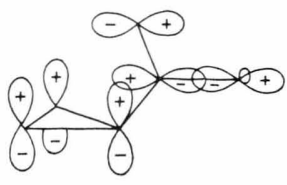

$\pi-\mathrm{MO}$
Fig. 2. Diagrams of the 4 highest MO's of cyclopropylmethyl ketone as calculated by the MINDO/2 method.

introduction. This finding may be attributed to the big MO coefficient of $\Delta_{\text {ext.as. }}$ and the small $\Delta_{\text {ext.s. }}$ MO coefficient at $\mathrm{C}_{1}$. The shapes of the MO's are similar to those of the cyclopropyl-methyl ketone.

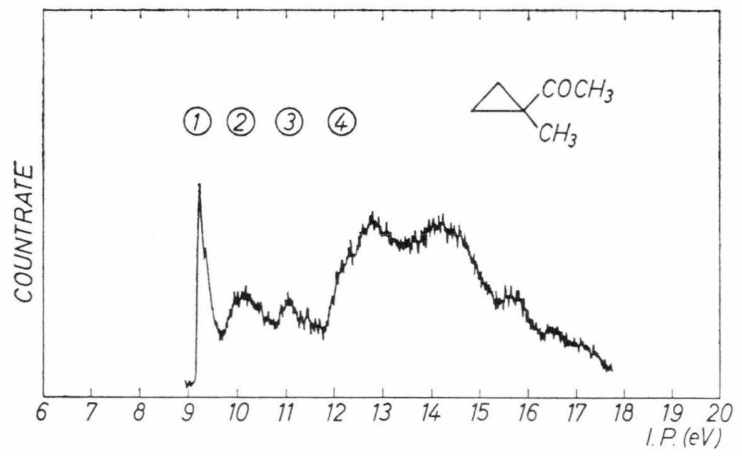

Fig. 3. PE spectrum of 1-methyl-cyclopropyl-methyl ketone.

Introducing a methyl group to the $\mathrm{C}_{2}$ atom af the ketone causes an inductive shift in the energies of all 4. MO's too. The new ionization energies and (MINDO/2) MO energies are: $\mathrm{n}-\mathrm{MO} 9.38 \mathrm{eV}(9.43$ $\mathrm{eV}), \Delta_{\text {ext.as. }} 10.13 \mathrm{eV}(10.18 \mathrm{eV}), \Delta_{\text {ext.s. }} 11.05 \mathrm{eV}$ $(10.22 \mathrm{eV})$ and $\pi$-MO $12.26 \mathrm{eV}(11.37 \mathrm{eV})$. These values, compared with those of 1-methyl-cyclopropyl-methyl ketone show a smaller shift for the $\Delta_{\text {ext.as. }}$ MO and a bigger shift for the $\Delta_{\text {ext.s. }}$ MO. The alteration in the magnitudes of the shifts is

1 M. Shanshal, Z. Naturforsch. 27 a, 1665 [1972].

2 E. Heilbronner, R. Gleiter, T. Hoshi, and A. de Meijere, Helv. 56, 1594 [1973].

3 J. A. Al-Khafaji and M. Shanshal, Z. Naturforsch. 30 a, 1023 [1975]. parallel to the size of the MO coefficients at $\mathrm{C}_{2}$. $\Delta_{\text {ext.s. }}$ has a bigger coefficient at $\mathrm{C}_{2}$ than the $\Delta_{\text {ext.as. }}$ MO (Figure 5). The MINDO/2 calculation results show a considerable delocalization of the oxygen lone pair orbital in the three membered ring. Similar but weaker inductive shifts are expected for this MO. In fact the PE spectra show an increase in the orbital energy of $0.16 \mathrm{eV}$ due to $\mathrm{C}_{1}$ methylation and only $0.08 \mathrm{eV}$ due to $\mathrm{C}_{2}$ methylation.

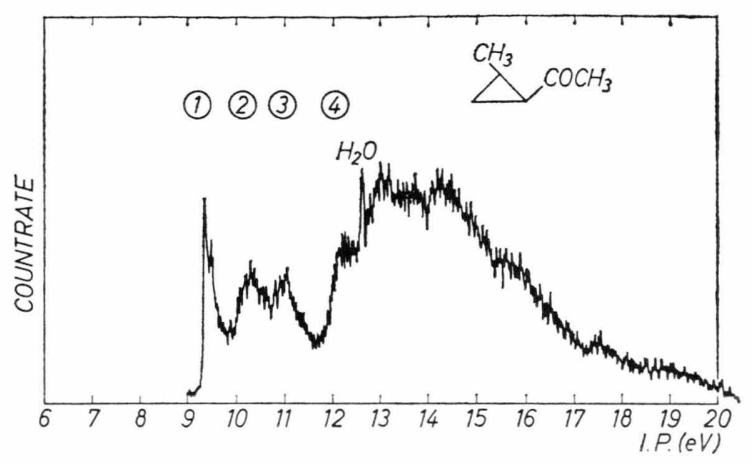

Fig. 4. PE spectrum of 2-methyl-cyclopropyl-methyl ketone.

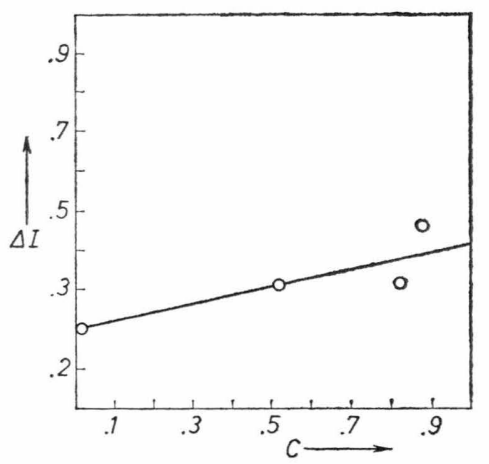

Fig. 5. Energy shifts of the Walsh MO's in methyl-cyclopropyl-methyl ketones vs. MO coefficients at the methylation site.

Acknowledgement. The authors thank Prof. Dr. R. Gleiter, T.H. Darmstadt, W.-Germany, for providing the possibility of PE spectral measurements.

4 A. Y. Mayer, B. Muel, and M. Kasha, J. Mol. Spectroscopy 43, 262 [1972].

5 T. Koopmans, Physica 1, 104 [1934].

6 See D. W. Turnar, C. Baker, A. D. Baker, and C. R. Brundle, Molecular Photoelectron Spectroscopy, Wiley Interscience, London 1970. 